\title{
Phosphorus-31 Nuclear Magnetic Resonance Analysis of Transient Changes of Canine Myocardial Metabolism In Vivo
}

\author{
F. W. Heineman and R. S. Balaban \\ With the technical assistance of Susan James \\ Laboratory of Cardiac Energetics, National Heart, Lung and Blood Institute, National Institutes of Health, Bethesda, Maryland 20892
}

\begin{abstract}
The time course of the relative myocardial phosphocreatine and adenosine triphosphate contents (PCr/ATP) during step changes in heart rate in vivo was studied in 14 dogs using ${ }^{31} \mathrm{P}$ nuclear magnetic resonance (NMR) to determine if transient changes in the high energy phosphates occur with changes in cardiac work. Coronary sinus blood flow (CF), oxygen consumption $\left(\mathrm{MVO}_{2}\right)$, and $\mathrm{NMR}$ data were simultaneously measured during brief $(\sim 3 \mathrm{~min})$, paced increases in heart rate in these open chest animals. ${ }^{31} \mathrm{P}$ spectra were collected with a time resolution of 15-16 s ( $\mathrm{PCr}$ signal to noise 22-41:1). Paced tachycardia associated with increased $\mathrm{CF}$ and $\mathrm{MVO}_{2}$ had no significant transient or sustained effect on PCr/ATP. Higher heart rates, associated with decreased $\mathrm{CF}$ and blood pressure, caused rapid decreases of PCr/ATP that were reversible upon return to control rates. These data indicate that there are no transient changes in ${ }^{31} \mathrm{P}$ metabolites (on a 15-16-s time base) during step changes in cardiac work associated with increased CF. This lack of change demonstrates that ATP hydrolysis and production are closely matched and that the feedback mechanism linking these processes occurs rapidly with no detectable transient change in the phosphate metabolites. In contrast, when the CF response to tachycardia is insufficient $\mathrm{PCr}$ is quickly depleted. This latter result suggests that the PCr/ATP ratio may be a sensitive, rapidly responding indicator of coronary supply/demand mismatching in vivo. (J. Clin. Invest. 1990. 85:843-852.) oxygen consumption • ischemia • energy • metabolism • adenosine triphosphate $\bullet$ creatine phosphate
\end{abstract}

\section{Introduction}

Adenosine triphosphate (ATP) is the major intermediary between the metabolic energy conversion processes (i.e., glycolysis and oxidative phosphorylation) and the contractile elements (i.e., myofibrils) in the heart. As such, the variations in the high energy phosphate compounds have been used as an index of the balance between myocardial energy consumption and supply. With the recent application of ${ }^{31} \mathrm{P}$ nuclear magnetic resonance (NMR) ${ }^{1}$ techniques it is now feasible to follow

Address reprint requests to Dr. Heineman, Laboratory of Cardiac Energetics/NHLBI, National Institutes of Health, 9000 Rockville Pike, Building 1/B3-07, Bethesda, MD 20892.

Received for publication 14 February 1989 and in revised form 24 August 1989

1. Abbreviations used in this paper: $\mathrm{CF}$, coronary sinus blood flow; FID, free induction decay; MAP, mean arterial blood pressure; $\mathbf{M V O}_{2}$, myocardial oxygen consumption; NMR, nuclear magnetic resonance: $\mathrm{RPP}$, rate pressure product; $\mathrm{S} / \mathrm{N}$, signal to noise ratio.

The Journal of Clinical Investigation, Inc.

Volume 85, March 1990, 843-852 the concentrations in vivo of these important intermediates of energy metabolism (1).

Previous studies using ${ }^{31} \mathrm{P}$ NMR have provided valuable information on the metabolic effects of myocardial hypoxia, ischemia, and work (2-4). One such finding was that the concentrations of high energy phosphate compounds and their metabolites do not change significantly over a wide range of steady state cardiac workloads $(5,6)$. This observation was particularly surprising considering the widely held view that the rate of mitochondrial oxidative phosphorylation is modulated by variations in the concentrations of the ATP hydrolysis products, namely inorganic phosphorus $\left(P_{i}\right)$ and $\operatorname{ADP}(7,8)$. The lack of detectable change of the high energy phosphate compounds and $P_{i}$ despite steady state increases in the rate of myocardial oxygen consumption $\left(\mathrm{MVO}_{2}\right)$ implies that ADP and $P_{i}$ are not the primary regulators of mitochondrial respiration in vivo. However, no data on the transient, in vivo behavior of these metabolites immediately after a change in cardiac work are currently available, nor is there information on the time course of the high energy phosphate compounds in response to workloads exceeding the physiologic reserves of the heart. Computer simulation studies have predicted transient changes in the myocardial concentrations of phosphate compounds over a 1-3-min time course after a sudden increase in cardiac work (9). Previous ${ }^{31} \mathrm{P}$ NMR studies have either intentionally focused on periods of stable myocardial metabolism or have lacked sufficient time resolution to detect these predicted transient changes.

The purpose of this study was to use time-resolved ${ }^{31} \mathrm{P}$ NMR spectroscopy (resolution 15-16 s) to determine whether or not transient changes occur in myocardial phosphocreatine (PCr) and ATP over the course of rapid changes in cardiac work. This information is important in determining the mechanism of mitochondrial respiratory control during an increase in cardiac work. The time course of these measurements is compared to the simultaneously occurring changes in left ventricular blood flow and arterial blood pressure for workloads both within and exceeding the hemodynamic reserves of the heart. These studies also were conducted to evaluate the time course of the phosphate metabolite response to the supply/demand mismatch that occurs with high levels of artificially induced tachycardia.

\section{Methods}

Surgical preparation. 14 beagles of either sex (body weight $10-16 \mathrm{~kg}$ ) were sedated with $20 \mathrm{mg} / \mathrm{kg}$ thiopental i.v., intubated and ventilated using a servo-pediatric ventilator (900D; Siemens-Elema AB, Solna, Sweden). The gas mixture consisted of $80 \% \mathrm{O}_{2}, 0.7-1.0 \%$ halothane to maintain anesthesia and $\mathrm{N}_{2} \mathrm{O}$ balance. Respiratory rate was set at $\sim 0.5 \mathrm{~Hz}$ and changes in minute volume, when indicated by arterial blood gas measurements, were made by varying the tidal volume. Aortic pressure and arterial blood gases were measured from a cannula advanced into the aorta via the left carotid artery. The cannula was 
connected to a solid state, nonmagnetic transducer (Cobe Laboratories, Inc., Lakewood, $\mathrm{CO}$ ) located immediately outside of the magnet. A median sternotomy was performed and all pericardial fat was removed by blunt dissection and low current cautery.

In nine dogs, heparin (initial dose $2000 \mathrm{U}$ ) was administered intravenously. The ends of a shunt of Tygon tubing ( $1 / 8$ inch i.d., $1 / 4$ inch o.d. length $35 \mathrm{~cm}$; Norton Performance Plastics, Akron, $\mathrm{OH}$ ) were passed from the left jugular vein into the coronary sinus and from the right jugular vein into the right atrium. A suture (3-0 Tevdek II, noncutting needle; Pfizer Hospital Group, Inc., Queens Village, NY) was placed around the coronary sinus near its junction with the right atrium and tied over the tubing. Thus, coronary sinus blood flow was diverted through the extracorporeal shunt and returned to the right atrium. The shunt contained a flow probe (Transonics Systems Inc., Ithaca, NY) and a port for sampling the coronary sinus blood (10). Hydration was maintained with Ringer's solution given through a peripheral venous cannula. Bipolar pacing leads were placed on the surface of the right atrium through a small incision in the pericardium. A two-turn, home-built surface coil $(3 \mathrm{~cm}$ o.d.) was sutured to the pericardium overlying the anteroseptal portion of the left ventricle as previously described (11). The animals were then placed on a circulating warm water blanket in a rigid plastic cradle that permitted centering of the heart in the $x-y$ axes. The thoracotomy was sealed with plastic wrap to reduce heat and water loss and the entire preparation was wrapped in a heat reflective "space blanket" (Boy Scouts of America, Charlotte, NC). In previous studies, we have found that this technique maintained the animals body temperature at $37 \pm 0.5^{\circ} \mathrm{C}(11)$. The animal and cradle were moved into the NMR magnet without interrupting the positive pressure ventilation and positioned to center the heart along the $z$ axis of the magnetic field. The remaining five dogs were not heparinized and a coronary sinus shunt was not placed, but otherwise underwent the same protocol as the animals with shunts. Hardcopies of blood pressure, heart rate, and coronary sinus flow were made on a Gould 2600S recorder (Gould, Inc., Cleveland, $\mathrm{OH}$ ), along with a digital pulse from the NMR spectrometer to verify the timing of data acquisitions relative to the cardiac cycle. All protocols were approved by the Intramural NHLBI Animal Care and Use Committee (protocol 6-KE-1).

NMR measurements. All experiments were conducted in a 4.7 Tesla $26 \mathrm{~cm}$ horizontal clear bore magnet (Oxford Instruments, Oxford, UK) equipped with a CSI spectrometer (General Electric Corp., Freemont, CA). The surface coil was tuned to $81 \mathrm{MHz}$ and matched to $50 \mathrm{ohms}$. All pulse sequences were triggered from the ventilator digital pulse at the start of inspiration. The heart was paced at a precise harmonic of the respiratory rate synchronized to the ventilator using a laboratory computer (Digital MINC 11/73) (11). Although it has been previously demonstrated that the high energy phosphate compounds do not change significantly over the time course of the cardiac cycle $(12,13)$, NMR data acquisitions at all heart rates were timed to occur during the first diastole at the beginning of a respiratory cycle to minimize motion artifacts. The field was manually shimmed using the proton free induction decay (FID) at $200 \mathrm{MHz}\left({ }^{1} \mathrm{H}\right.$ linewidth $36-48$ $\mathrm{Hz}) .{ }^{31} \mathrm{P}$ spectra were collected using a simple one pulse and collect method with a pulse width of 40-50 $\mu \mathrm{s}$ and an interpulse delay of $\sim 2 \mathrm{~s}$ (determined by the precise respiratory rate). This pulse width choosen in each animal as providing the maximum $\mathrm{PCr}$ signal to noise for the interpulse delay used (11). Before and after the experimental protocol, reference spectra of 120-200 acquisitions were collected for use with the line fitting routine (described later) and to check the stability of the ${ }^{31} \mathrm{P}$ spectrum.

Pacing protocol. Paced tachycardia allows rapid initiation and reversal of the change in cardiac work and its effects are largely limited to the heart as long as arterial blood pressure is maintained. Even if central compensatory responses tend to restore the control blood pressure, the rate pressure product and $\mathrm{MVO}_{2}$ remain elevated due to the tachycardia. In addition to being useful as a means of increasing cardiac work, paced tachycardia may be taken to a level that produces cardiac decompensation $(14,15)$ and/or supply/demand mismatching in both clinical and research settings, without producing irreversible myocardial damage. From preliminary observations it was decided to use paced changes in heart rate as a means of producing step increases and decreases in $\mathrm{MVO}_{2}$.

Cardiac pacing was synchronized to the respiratory cycle by driving the pacemaker from a PDP 11/23 computer (Digital Equipment Corp., Maynard, MA) at harmonics (accurate to $<1 \mathrm{~ms}$ ) of the respiratory rate. The BASIC program for performing this task is available from the authors. Heart rate for the control periods was set at the lowest harmonic of the respiratory rate (i.e., $\sim 30$ breaths $/ \mathrm{min}$ ), which exceeded the unpaced rate of the animal. A 120-200 acquisition (4-7 min) control spectrum was acquired, after which harmonic step increases of the heart rate were performed lasting $\sim 3$ min with intervening $\sim 3$ min returns to the control rate. Heart rate was increased in this manner until a rate was attained where the coronary sinus flow decreased (rather than increased) or pacing capture could no longer be maintained. In the five dogs without shunts heart rate was increased in the same manner until MAP decreased or capture could not be maintained. In the dogs with shunts, arterial and coronary sinus blood was sampled during each control and pace-up period and the oxygen content determined using a Lex- $\mathrm{O}_{2}$-Con (Hospex, Chestnut Hill, MA). ${ }^{31} \mathrm{P}$ NMR spectra were collected over 15-16-s blocks (eight acquisitions/ spectrum) continuously during the pacing protocol. After completing the sequence of pace-up and control periods, the heart rate was kept at the control rate while a postprotocol $120-200$ acquisition spectrum was accumulated.

Data analysis. NMR FIDs were baseline corrected using the baseline correction routine resident in the GEMCSI software, zero-filled to 2 Kbytes and exponentially filtered to provide optimal line-broadening for phosphocreatine ( $\mathrm{PCr}$ linewidth was between 7 and $15 \mathrm{~Hz}$ ) before Fourier transformation. PCr/ATP for each spectrum was calculated as the ratio of the $\mathrm{PCr}$ and $\beta$-ATP peak areas using a least-squares fitting routine written for the Nicolet 1280 computer in the CSI system (16). Briefly, a high signal-to-noise reference spectrum was used as a model of the lineshape for each peak and fit on a least squares, point-by-point basis to the lower signal-to-noise data spectra. This method is more precise, at least as accurate, and requires far less time $(\sim 1 \mathrm{~s}$ to measure two peak windows in a 2-Kbyte spectrum) than conventional methods such as integration or Lorentzian/Gaussian fitting.

$\mathrm{PCr} / \mathrm{ATP}$ ratios at the highest heart rate associated with an increase in hemodynamic parameters (coronary sinus flow and arterial blood pressure) were normalized to the control period (to compensate for variation between animals) and tested by one-way ANOVA for differences over the entire time course of each step-up and step-down in heart rate. If significant differences were found to exist, individual a posteriori comparisons were made between the PCr/ATP values for the control period versus each subsequent time point by $t$ test. A Bonferroni correction factor was applied to the $P$ values from the $t$ tests to compensate for the multiple comparisons. The same statistical analyses were applied to the $\mathrm{PCr} / \mathrm{ATP}$ ratios at heart rates associated with a decrease in coronary sinus flow or arterial blood pressure. A $P$ value $<0.05$ was considered significant in all statistical tests. Since the value of $\beta$ (for a type II error) is not available, the results of statistical tests where a lack of significance was of specific interest are given as the calculated $P$ value. Normality of the data distributions and homogeneity of variances were tested using the Wilk-Shapiro and $F$-max tests, respectively, before performing two-tailed $t$ tests or ANOVA. The large majority of data met the requirements for ANOVA or $t$ tests. The exceptions were analyzed using the Wilcoxon signed rank or the Mann-Whitney nonparametric procedures. All descriptive data are presented as the mean $\pm \operatorname{SEM}(17,18)$.

Mean arterial blood pressure (MAP) was calculated from the pressure waveform as diastolic $+1 / 3$ pulse pressure and used to calculate the rate pressure product ( $R P P=$ heart rate $\times$ MAP). Left ventricular oxygen consumption $\left(\mathrm{MVO}_{2}\right)$ was estimated as the product of the coronary sinus flow (CF) and the arterial-coronary sinus $\mathrm{O}_{2}$ content difference. MAP, CF, RPP, and $\mathrm{MVO}_{2}$ for the control and pace-up periods were compared by two-way ANOVA (17). 
Table I. Hemodynamic Parameters in Dogs with Coronary Sinus Shunts

\begin{tabular}{|c|c|c|c|c|c|c|}
\hline & BPM & MAP & CS flow & $\mathrm{MVO}_{2}$ & RPP & $n$ \\
\hline Initial & $99(3)$ & $82(6)$ & $20.5(1.4)$ & $1.45(0.27)$ & $8,124(514)$ & 5 \\
\hline Pace-up A & $157(11)$ & $88(9)^{*}$ & $26.6(1.4)^{*}$ & $2.52(0.65)^{*}$ & $13,518(1,174)^{*}$ & 5 \\
\hline Post & $99(3)$ & $81(6)$ & $19.6(1.6)$ & $1.38(0.32)$ & $8,064(484)$ & 5 \\
\hline Initial & $113(7)$ & $75(1)$ & $22.2(0.9)$ & $1.59(0.22)$ & $8,197(322)$ & 8 \\
\hline Pace-up B & $198(7)$ & $46(1)^{*}$ & $17.0(0.9)^{*}$ & $1.29(0.27)$ & $8,111(706)$ & 8 \\
\hline Post & $113(7)$ & $66(2)^{*}$ & $27.8(1.9)^{*}$ & $1.43(0.19)$ & $7,839(292)$ & 8 \\
\hline
\end{tabular}

Abbreviations: BPM, paced heart rate in beats per minute; CS flow, blood flow through the coronary sinus shunt in milliliters/minute; $\mathrm{MVO}_{2}$, myocardial oxygen consumption in micromoles/gram of left ventricle/minute. Pace-up A represents values for tachycardias associated with increases in $\mathrm{MVO}_{2}>50 \%$. Pace-up B represents values for tachycardias associated with a decrease in flow and MAP. All values expressed as mean (SEM). * Statistical significance at $P<0.05$.

The five dogs without coronary sinus shunts were used to ascertain whether or not the shunt influenced the outcome of the experiments. The NMR and hemodynamic data from these animals were analyzed in the same manner as were the data for the dogs with shunts. In addition, the PCr/ATP ratios of the control and tachycardic periods in the dogs without shunts were compared by one-way ANOVA to the corresponding periods in the dogs with shunts.

\section{Results}

Hemodynamic data. Hemodynamic parameters (heart rate, arterial blood pressure, RPP, CF) and $\mathrm{MVO}_{2}$ during the control periods (Tables I and II) were comparable to the corresponding measurements previously made in our laboratory on dogs $(6,11)$. The average control $\mathrm{MVO}_{2}$ in the animals with coronary sinus shunts (Table I) was $1.45 \pm 0.26 \mu \mathrm{mol} / \mathrm{g}$ per min $(3.71 \pm 0.67 \mathrm{ml} / 100 \mathrm{~g}$ per $\mathrm{min})$. Control $\mathrm{MVO}_{2}$ ranged from 0.50 to $3.00 \mu \mathrm{mol} / \mathrm{g}$ per min.

Coronary sinus flow approximates left ventricular blood flow, but it is neither the only route for venous return from the left ventricle nor is its content exclusively comprised of left ventricular venous blood. For these reasons we consider the blood flow and $\mathrm{MVO}_{2}$ measured in these experiments estimates of those for the left ventricle. A major concern with this model was the possible influence of the shunt on the hemodynamics of the coronary sinus. This effect was studied in pre-

Table II. Hemodynamic Parameters in Dogs without Coronary Sinus Shunts

\begin{tabular}{lcccc}
\hline & BPM & MAP & RPP & $n$ \\
\hline Initial & $96(3)$ & $79(1)$ & $7,602(486)$ & 3 \\
Pace-up A & $149(11)$ & $90(2)^{*}$ & $13,616(1,721)^{*}$ & 3 \\
Post & $96(3)$ & $77(1)$ & $7,408(481)$ & 3 \\
Initial & $110(11)$ & $81(2)$ & $9,121(1,563)$ & 5 \\
Pace-up B & $189(10)$ & $49(1)^{*}$ & $8,494(1,315)$ & 5 \\
Post & $110(11)$ & $78(2)$ & $9,004(1,599)$ & 5 \\
& & & &
\end{tabular}

Abbreviations, units, and statistics are the same as for Table I * $P<0.05$.

Pace-up A represents values for tachycardias associated with an increase in RPP > 50\%.

Pace-up B represents values for tachycardias associated with a decrease in MAP. liminary experiments using a shunt that contained a second, smaller lumen to directly monitor the coronary sinus pressure with the cannula in place. At basal rates of coronary sinus flow, the coronary sinus pressure remained below $4 \mathrm{mmHg}$ throughout the cardiac cycle. At rates of flow exceeding those occurring in the protocol studies the diastolic coronary sinus pressure remained $<6 \mathrm{mmHg}$ and systolic pressures reached a peak of $16 \mathrm{mmHg}$. This represents a worst case since, due to the second lumen, the diameter of the flow lumen of the twolumen shunt was $\sim 25 \%$ smaller than the cannula used during the protocol experiments. Coronary sinus pressure was not monitored during the protocol experiments so that the cannula lumen could be maximized, thus minimizing its resistance.

For reasons addressed in the Discussion, data for tachycardias associated with increased $\mathrm{MVO}_{2}$ were only included in this group if there was at least a $50 \%$ increase in $\mathrm{MVO}_{2}$ or, in the case of animals without shunts, a 50\% increase in RPP. Five of the nine dogs with shunts (mean $\mathrm{MVO}_{2}$ increase $78 \%$, range 64 to 112 per cent) and three of the five without shunts (mean RPP increase $82 \%$, range 51 to $105 \%$ ) met these criteria. Eight of the nine animals with shunts attained a level of tachycardia that produced a decrease in coronary sinus flow and five of the five animals without shunts reached a level of tachycardia causing MAP to decrease. To summarize, in the group of nine animals with a coronary sinus shunt: four dogs attained an increase in $\mathrm{MVO}_{2}>50 \%$ at one heart rate and also reached a higher rate that produced a fall in coronary flow; four dogs did not have a paced heart rate which caused $\mathrm{MVO}_{2}$ to increase $>50 \%$ but did reach a rate that resulted in decreased coronary flow; 1 dog reached a heart rate that produced an increase in $\mathrm{MVO}_{2}>50 \%$ but could not be paced to a rate causing coronary flow to decrease. Similarly, in the five dogs without shunts: three dogs attained heart rates at which RPP increased $>50 \%$ and also higher rates at which MAP decreased from the control level; two dogs did not have a paced harmonic heart rate which increased RPP by $>50 \%$ but could be paced to higher rates where MAP decreased from control levels.

Hemodynamic indices of the dogs that underwent the experimental protocol without the placement of a coronary sinus shunt (Table II) were comparable during the control periods, as well as during the periods of increased heart rate, to dogs with coronary sinus shunts (Table I). The heart rates at which increases or decreases in hemodynamic parameters were ob- 

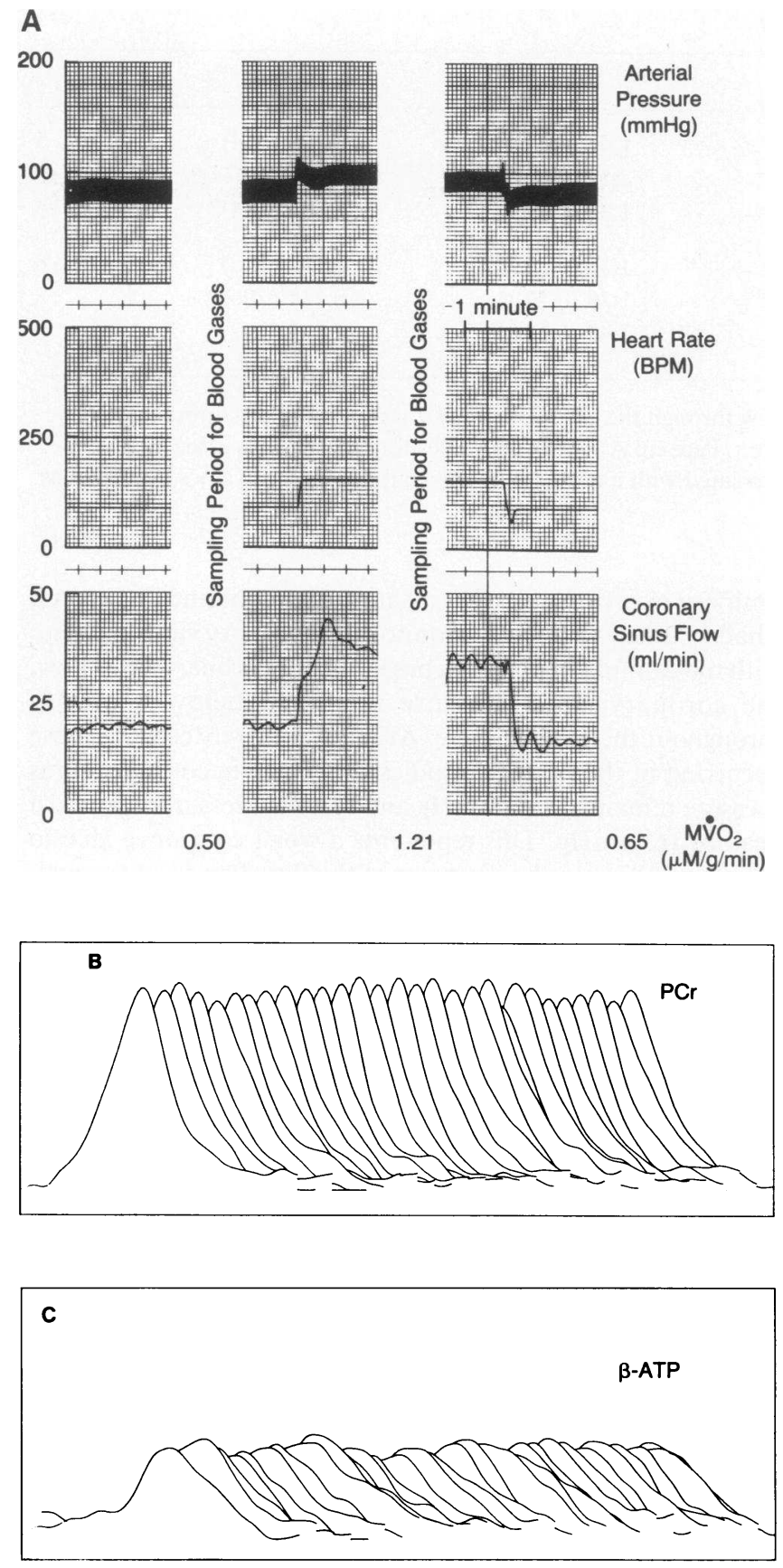

served as well as the relative number of animals that satisfied the criteria for $>50 \%$ increase in $\mathrm{MVO}_{2}$ or RPP were also similar between the shunt and no shunt groups.

${ }^{31} P$ NMR measurements. The status of myocardial high energy phosphate compounds in these experiments was expressed as the PCr/ATP ratio. Through the creatine kinase reaction (see Appendix, Eq. A2), the maintenance of the terminal phosphate of ATP is thermodynamically favored over that of PCr. Thus, ATP concentrations remain relatively constant at the expense of $\mathrm{PCr}$, until the metabolic stress (e.g., ischemia) becomes severe. For the same reason, $\mathrm{PCr}$ concentrations are inversely related to ADP (and $P_{i}$ ). Since ADP concentrations in normal tissue are too low to be directly measured by either NMR or conventional extraction techniques, the NMR peak of $\mathrm{PCr}$ is a useful indicator of changes in the ADP concentra-
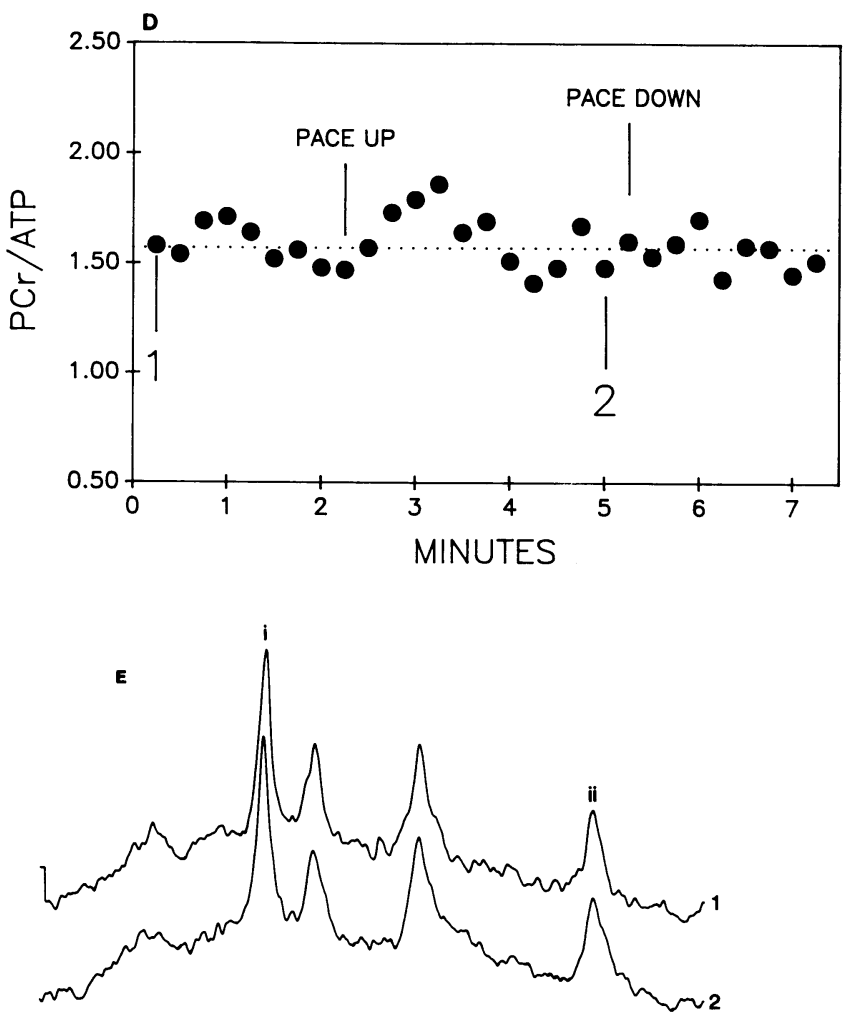

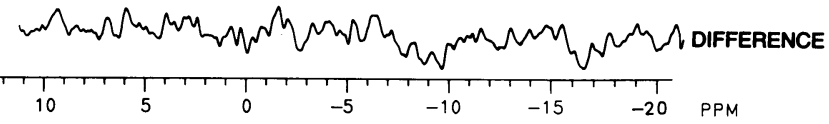

Figure 1. Sample hemodynamic and ${ }^{31} \mathrm{P}$ NMR time courses for a tachycardia producing an increase in $\mathrm{MVO}_{2}>50 \%$. $(A)$ Hemodynamic record. Recording was interrupted after blood pressure and flow had stabilized (at the points indicated) to sample coronary sinus and arterial blood. Values for myocardial oxygen consumption $\left(\mathrm{MVO}_{2}\right)$ during the stable periods are presented as micromoles/gram of left ventricle/minute. $(B)$ Stack plot of the phosphocreatine (PCr) resonant peaks recorded over the time course shown in $A$. Note the lack of change in the peak heights. $(C)$ Stack plot of the $\beta$-adenosine triphosphate (ATP) peaks recorded over the time course shown in $A$. Note the stability of the signal over the course of the tachycardia. $(D)$ Ratios of the PCr and ATP peak areas (uncorrected for differential saturation) over the time course shown in $A$. (E) Representative spectra from the control (1) and tachycardic (2) periods. Spectra 1 and 2 coincide with points 1 and 2 shown in $D .8$ pulses/spectrum, $14 \mathrm{~Hz}$ exponential line-broadening.

tion when expressed as a ratio with the area of a relatively stable peak, such as ATP (5). Consistent with this, the $\beta$-ATP resonant peak remained stable during tachycardias associated with either increased or decreased hemodynamic indices (Figs. 1 and 2).

For the sake of comparison to previous work, the estimated control intracellular ADP concentration was calculated from the creatine kinase equilibrium (Eq. A2). Using the values from the high signal-to-noise control spectra for intracellular pH (7.10, from the chemical shift of $\left.P_{i}\right)$ and for PCr/ATP of (1.86), and a value for myocardial ATP of $6.6 \mathrm{mM}$ and total creatine of $27.3 \mathrm{mM}(6,11)$, the calculated control [ADP] was $61 \mu \mathrm{m} /$ liter cell water. This is consistent with values of [ADP] previously reported for canine myocardial cells (6).

Peak-to-peak signal-to-noise (S/N) of $\mathrm{PCr}$ for the eight scan 

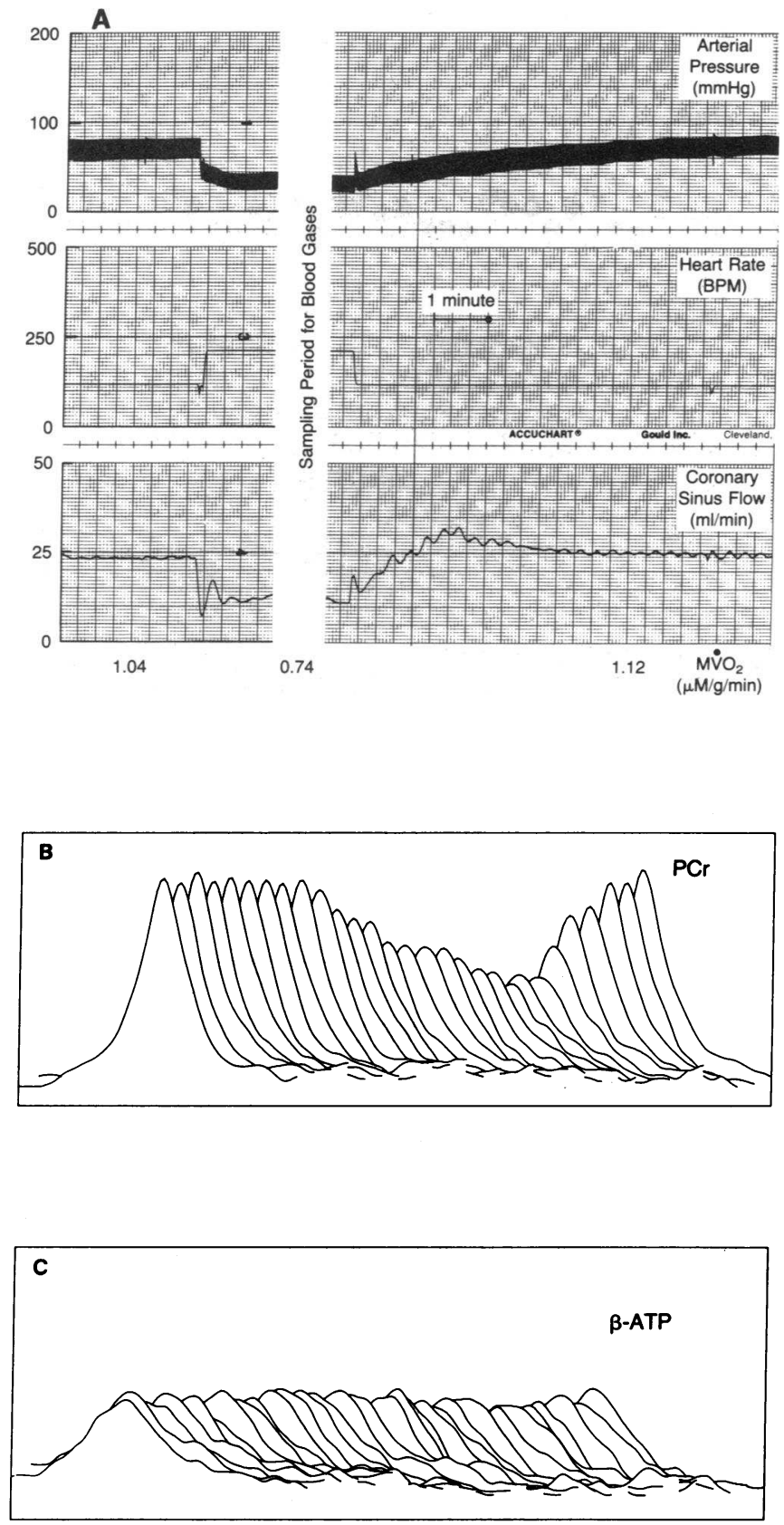

data spectra acquired over the time course of the pacing protocol averaged $30 \pm 1.6$ (range 22-41). Reference spectra (120-200 scans) recorded before and after the pacing protocol for use with the line fitting program had $\mathrm{PCr} \mathrm{S} / \mathrm{N}$ consistently greater than 140:1 (Fig. 3). The mean PCr/ATP for the control periods was $1.55 \pm 0.15$, uncorrected for differential saturation. The corrected value is $1.86 \pm 0.18$, which compares favorably with previously published values for the in vivo canine heart (6). All other PCr/ATP values in this study are expressed in terms of the ratio uncorrected for the effects of differential saturation. $\mathrm{PCr} / \mathrm{ATP}$ in dogs with a coronary sinus shunt did not significantly change $(P>0.86)$ from control values at any time during the pace-up associated with increased coronary sinus flow and $\mathrm{MVO}_{2}$ (Fig. 4). Additionally, there was no evidence of ischemia during these tachycardias judging from the
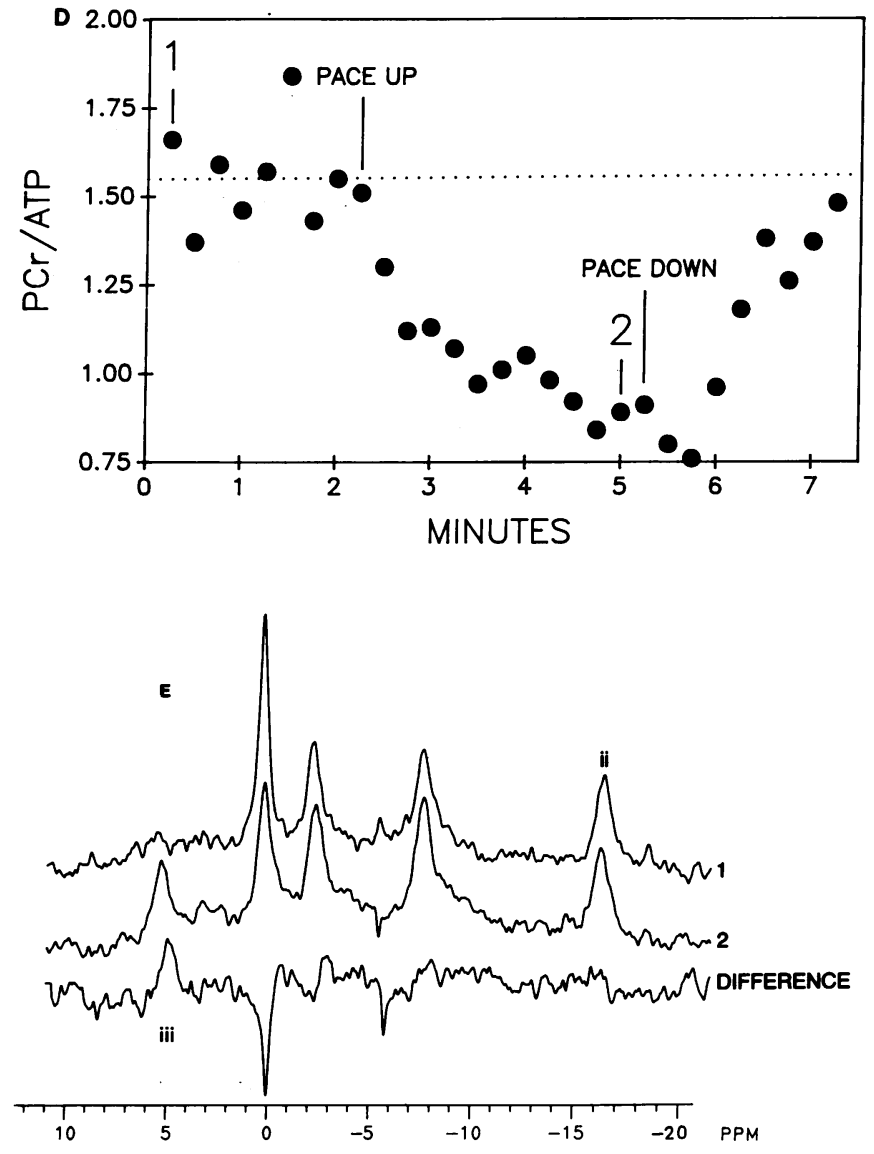

Figure 2. Sample hemodynamic and ${ }^{31} \mathrm{P}$ NMR time courses for a tachycardia producing a decrease in myocardial flow. See Fig. 1 legend for more detailed explanations of $A-E$. $(A)$ Note the rapid decrease in both flow and pressure after the initiation of the tachycardia and the hyperemic period following resumption of the control heart rate. (B) PCr peak height associated with the time course in $A$, illustrating the fall in $\mathrm{PCr}$ after the start of tachycardia and return to control levels after resuming control heart rate. $(C)$ Stack plot of $\beta$-ATP peaks coinciding with the $\mathrm{PCr}$ peaks in $B$ showing the relative stability of ATP during the tachycardia. (D) Simultaneous PCr/ATP ratios for the time course in $A-C$. $(E)$ Control and tachycardic spectra from points 1 and 2 in $D$. The tachycardia caused a decrease in $\operatorname{PCr}(a)$, no change in $\beta$-ATP $(b)$ and an increase in inorganic phosphorus $(c)$, evident in the difference spectrum. 8 pulses/spectrum, 12 $\mathrm{Hz}$ exponential linebroadening.

lack of a hyperemic phase and the prompt return of the MAP to the control values.

Conversely, paced tachycardia associated with decreased coronary sinus flow and no increase in $\mathrm{MVO}_{2}$ caused an immediate decline in PCr/ATP, which became statistically significant within $1 \mathrm{~min}$ and reached a plateau $\sim 2 \mathrm{~min}$ after the onset of the tachycardia (Fig. 5). Signal-to-noise of the 15-16-s spectra was not sufficient to accurately define either the magnitude or chemical shift of the $P_{i}$ peak. However, by averaging the spectra after PCr/ATP had reached a plateau following the onset of the paced tachycardia, it was possible to estimate the intracellular $\mathrm{pH}$ (from the chemical shift of $\mathbf{P}_{\mathrm{i}}$ ) during the period of decreased coronary flow as $7.13 \pm 0.05$. This did not differ significantly from the control $\mathrm{pH}(7.10)$ measured from the high signal-to-noise spectra $(P>0.20)$ and, even if it repre- 

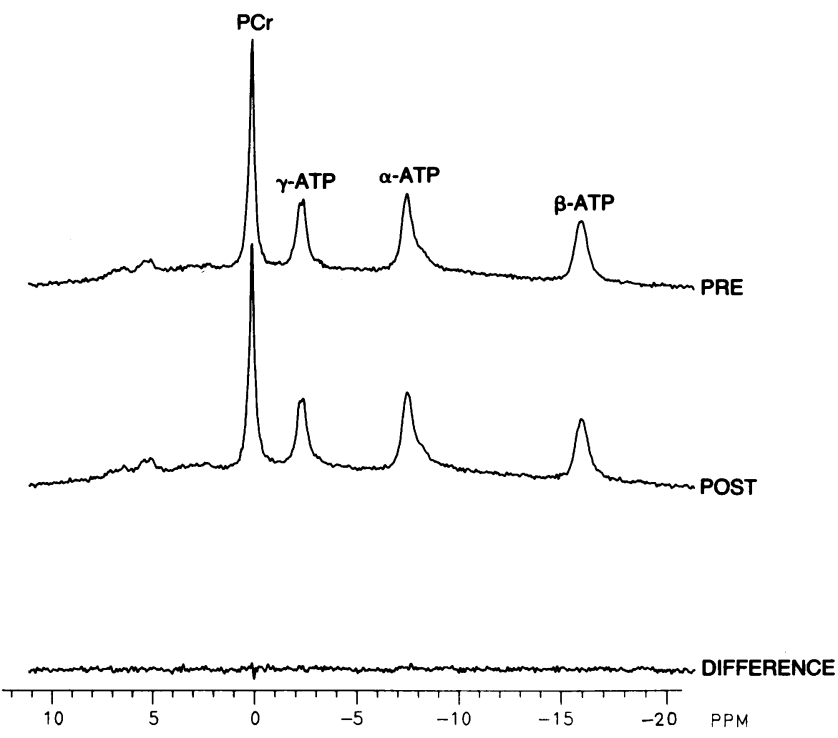

Figure 3. Control Spectra. Sample spectra acquired before (PRE) and after (POST) the pacing protocol, along with their difference, showing no appreciable change in the phosphocreatine $(\mathrm{PCr})$ or adenosine triphosphate ( $\alpha, \beta$ and $\gamma$-ATP) resonances. 200 pulses/spectrum, 2 $\mathrm{Hz}$ exponential line-broadening.

sents a real change, would affect $\mathrm{PCr}$ through the creatine kinase equilibrium (Eq. A2) $<5 \%$ in the opposite direction of the changes seen with pacing-induced decreases in coronary flow. The lack of change of intracellular $\mathrm{pH}$ during this brief stress is not surprising in view of the high buffer capacity of the myocardial cells (see reference 11). The decrease of PCr/ATP was fully reversible within $\sim 1$ min of resuming the control heart rate. Although there was a period of significant reactive hyperemia after this stress, there was no associated overshoot of the PCr/ATP ratio. MAP required $\sim 2 \mathrm{~min}$ to reach control levels following resumption of the control heart rate, as reflected by the significantly lower average MAP for the posttachycardia period (Table I). Sample experimental records of hemodynamic changes, the associated PCr/ATP ratios and sample spectra from control, pace up and post-control periods for both levels of tachycardia are presented in Figs. 1 and 2.

Dogs without coronary sinus shunts had control PCr/ATP ratios $(1.60 \pm 0.03)$, which did not statistically differ $(P>0.31)$ from those with shunts. In these animals tachycardias associated with increased blood pressure and RPP resulted in no change in PCr/ATP $(P>0.99)$. When the tachycardia in these dogs produced a significant decrease in the MAP and no change in the RPP the PCr/ATP ratio decreased significantly. These findings in dogs without shunts parallel those in dogs with shunts and demonstrate that the observed effects were independent of the cannulation of the coronary sinus.

Also important in demonstrating the validity of this model was the occurrence of a decrease in the PCr/ATP ratio with tachycardia associated with a blunted hemodynamic and metabolic response. In addition to demonstrating that PCr/ATP decreases when the $\mathrm{MVO}_{2}$ fails to increase despite tachycardia, this finding establishes that the sensitivity and time resolution of the NMR measurements were adequate to detect the predicted (but not observed) decrease of PCr/ATP for levels of tachycardia associated with an increase in $\mathrm{MVO}_{2}$. This is par-
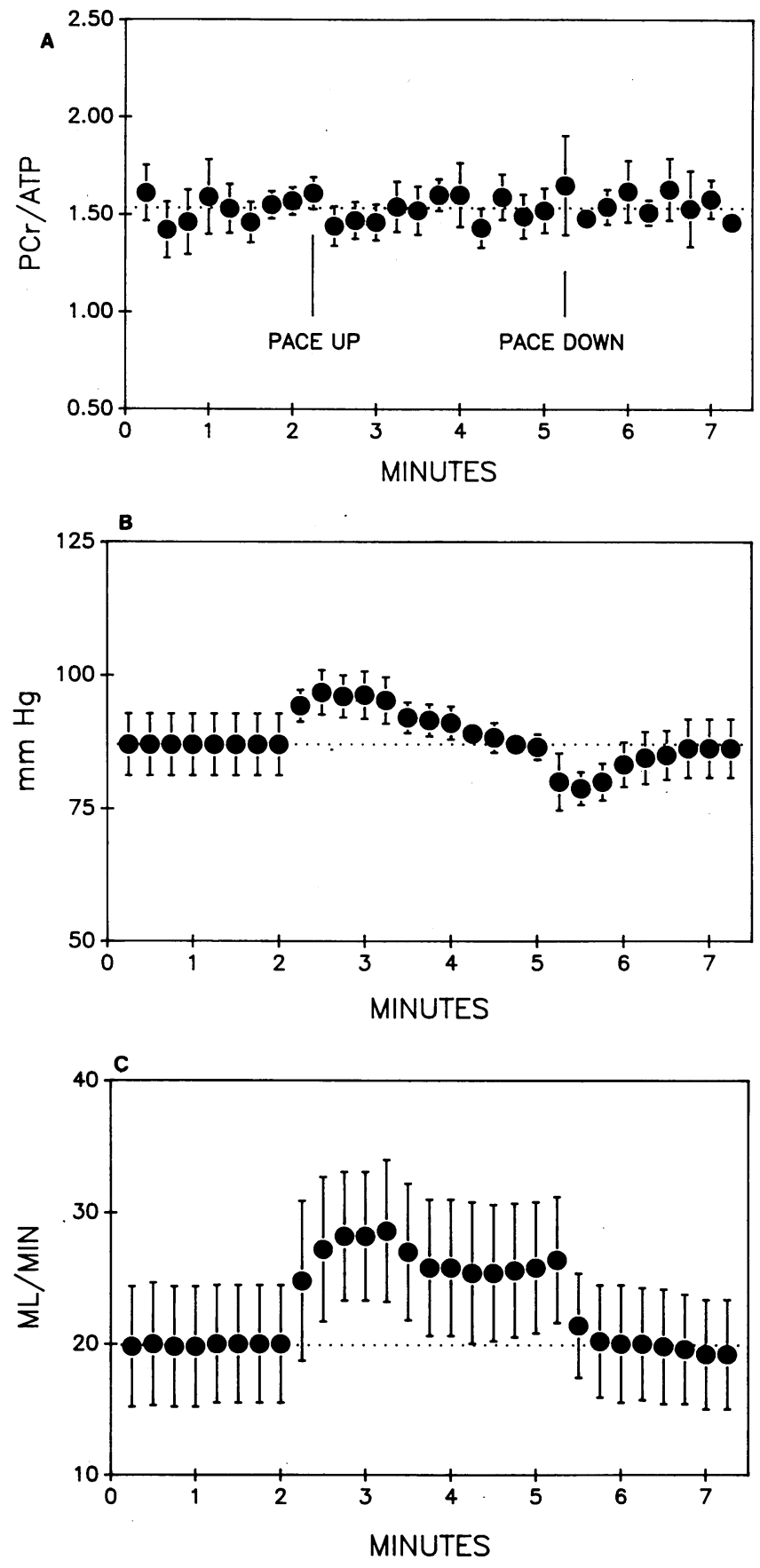

Figure 4. Simultaneous time courses of PCr/ATP, blood pressure and myocardial flow during a paced increase and decrease in heart rate associated with $>50 \%$ increase in $\mathrm{MVO}_{2}$. All values are means \pm SEM. Error bars along the time (minutes) axis are smaller than the symbol size $(<4 \mathrm{~s})$. All points represent data from five dogs. (A) Phosphocreatine/adenosine triphosphate (PCr/ATP) time course. Values not corrected for effects of differential saturation. Data presented are not normalized to illustrate the variability of the raw data. No significant differences were found to exist between the initial control period and the subsequent tachycardia and posttachycardia periods. (B) MAP in $\mathrm{mmHg}$. (C) Flow through the coronary sinus shunt in milliliters/minute.

ticularly important since the lack of statistical significance would be meaningless if using a measurement technique too insensitive to detect changes of the magnitude predicted. 

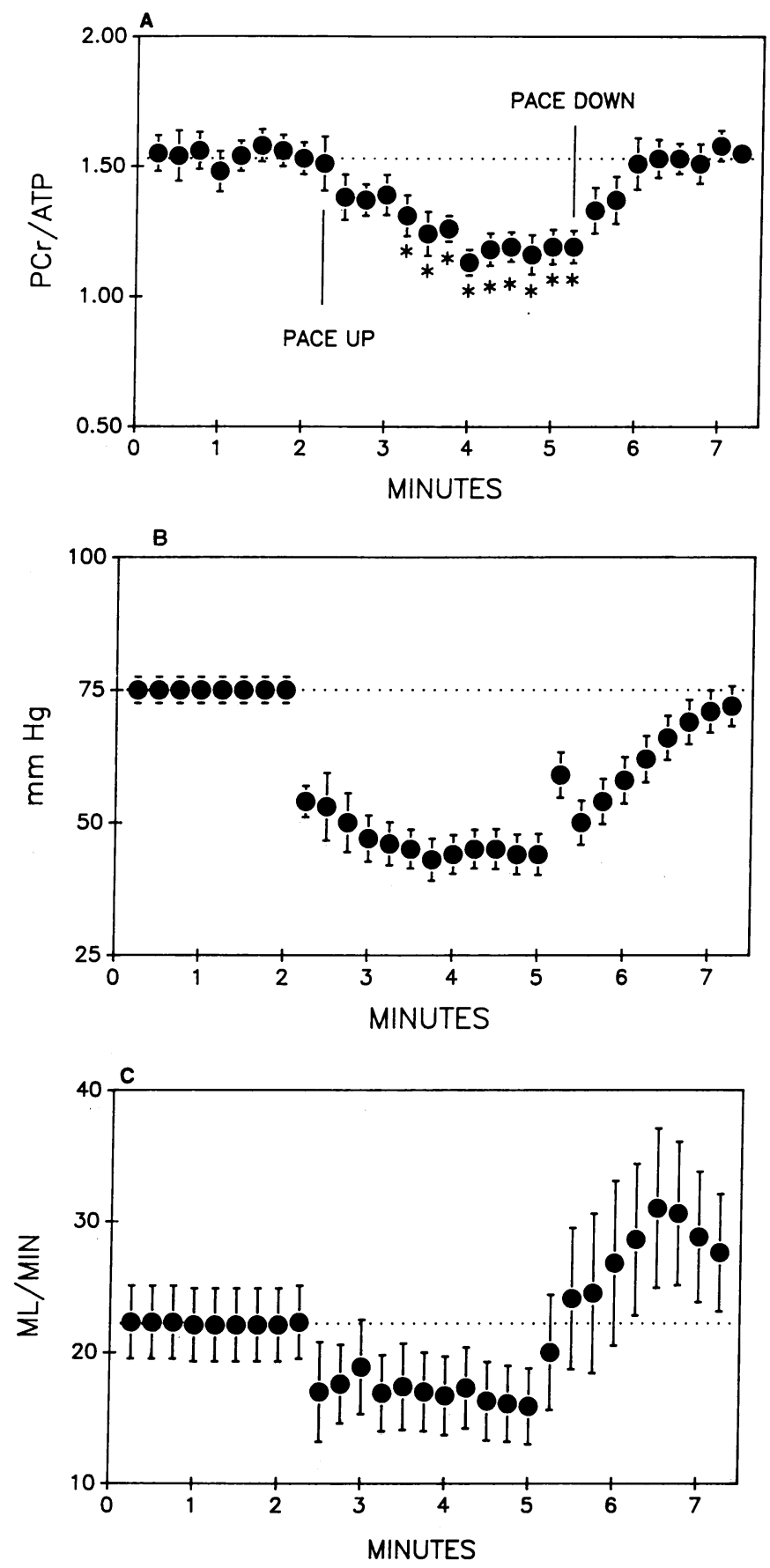

Figure 5. Simultaneous time courses of PCr/ATP, blood pressure and myocardial flow during a paced increase and decrease in heart rate associated with a decrease in myocardial flow. (See Fig. 4 legend). All points represent data from eight dogs. $(A)$ As in Fig. 4. ${ }^{*} \mathrm{De}-$ notes statistical difference from the control period $(P<0.05)$. ( $B$ and C) As in Fig. 4.

\section{Discussion}

An advantage of NMR as a tool for studying in vivo biochemistry is its ability to record serial measurements from the same region of living tissue and, thus, follow changes over the course of a metabolic event. Previous work using ${ }^{31} \mathrm{P}$ NMR has questioned the role played by phosphate metabolites in the regula-

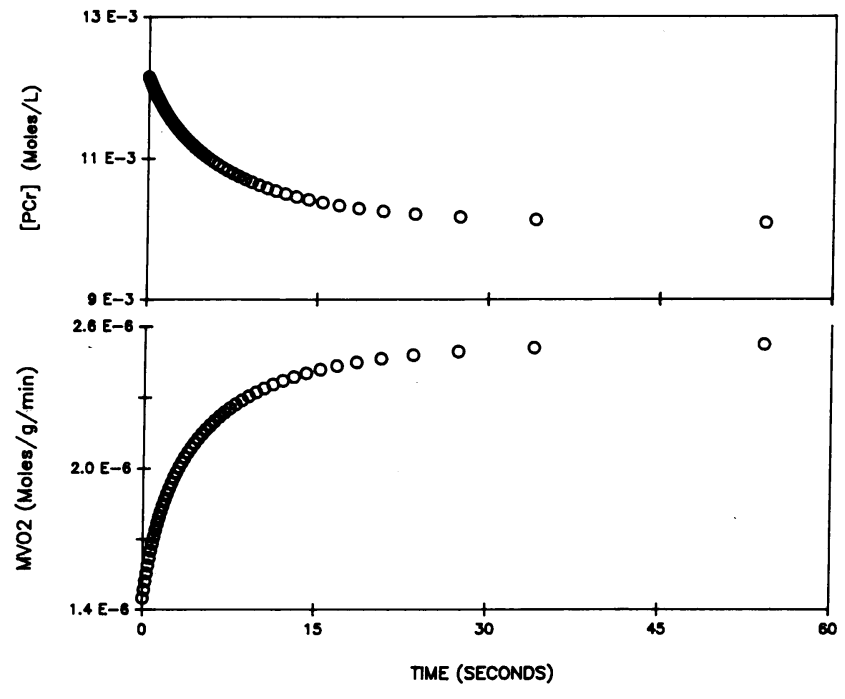

Figure 6. Calculated time courses of the changes in $\mathrm{MVO}_{2}$ and $\mathrm{PCr}$. Predicted change in myocardial oxygen consumption $\left(\mathrm{MVO}_{2}\right)$ in moles/gram of tissue/minute and PCr in moles/liter of cell water following a step increase in the rate of ATP hydrolysis at time zero. The boundary conditions were taken from the average values of $\mathrm{MVO}_{2}$ and $\mathrm{PCr}$ for the animals described in Table I and Fig. 4 and were used to solve for the transient phase (see Appendix).

tion of myocardial oxidative phosphorylation $(5,6)$. The current study extends this earlier work, focusing on the time course of the ${ }^{31} \mathrm{P}$ metabolites during the period immediately after rapid transients of cardiac metabolism which either fall within the functional reserve of the heart or exceed it.

We chose paced step changes between different heart rates to be the most rapid, reversible means of producing sudden transients in myocardial metabolism for the reasons discussed in Methods. Pacing has been previously established as means of inducing changes of $\mathrm{MVO}_{2}$ in both research and clinical settings. Paced tachycardia does not, however, produce increases in $\mathrm{MVO}_{2}$ or RPP as large as can be induced by other methods, such as $\beta$-agonist stimulation $(19,20)$. This limitation is secondary to decreased ventricular filling associated with artificial tachycardia, thoracotomy, anesthesia, and the nonphysiologic position of the animals (in this case, supine) which ultimately leads to a decline in hemodynamic performance (14). Furthermore, the NMR gating scheme used in this study to minimize motion artifacts only allowed the heart rate to be incremented as integer multiples of the respiratory rate, precluding the optimization of heart rate to maximize $\mathrm{MVO}_{2}$. From the results of previous work in this laboratory (6) it was felt that a change in $\mathrm{MVO}_{2} \geq 50 \%$ was a conservative estimate of the increase needed to elicit changes in PCr if ADP and $P_{i}$ serve as the regulators of oxidative phosphorylation. Even if the data for tachycardias associated with increased $\mathrm{MVO}_{2}$ from all nine animals with shunts were included in the statistical analyses, the outcome was the same. However, doing so decreases the chance of detecting a significant difference had PCr/ATP decreased. It was possible to find heart rates at integer multiples of the respiratory rate in five of the nine dogs with shunts which satisfied this criterion. In these animals $\mathrm{MVO}_{2}$ increased an average of $78 \%$. Using a simple model of respiratory control by $A D P$ and $P_{i}$ where $A D P$ and $P_{i}$ affinities 
are independent from each other (i.e., bireactant) (see Appendix), a 78\% increase in myocardial $\mathrm{MVO}_{2}$ would result in a decrease in the PCr/ATP ratio from the control value of 1.55 to 1.24 if the ATP hydrolysis products were the sole mechanism responsible for metabolic feedback to the mitochondria. This did not occur, as can be clearly seen in Fig. 4. A change of this magnitude would be statistically detectable by the methodology used in this study, as illustrated by the observation of a significant decrease in PCr/ATP among dogs paced to the point of hemodynamic compromise (Fig. 5). Moreover, the bireactant model is a worst case scenario. If the ATP hydrolysis products were not equally active in altering $\mathrm{MVO}_{2}$ the predicted change in PCr/ATP would be even greater.

It is evident from Fig. 4 that the PCr/ATP ratio during tachycardias associated with increased hemodynamic indices did not change sufficiently to account for the observed increase of myocardial respiration, confirming previous results conducted under steady state conditions $(6,21)$. The current study strengthens this premise, demonstrating that the lack of change of the PCr/ATP ratio is not simply due to a lack of time resolution or failure to observe a transient change after an increase in cardiac work. The lack of significant change in PCr/ATP over the entire time course of a step-up/step-down in heart rate (Fig. 4) also demonstrates that the metabolic feedback between the cytosolic use and mitochondrial production of ATP matches these two processes rapidly (i.e., within the 15-16-s time resolution of this study). If this were not the case the PCr/ATP ratio should have decreased during the initial stage of tachycardia (Fig. 4) until the production of ATP was increased to match the new demand. These observations support a mechanism of feedback for the regulation of myocardial respiration which is not primarily based on the concentrations of ADP and $P_{i}$. It should be noted that this differs from recent observations in the neonatal heart, where respiratory regulation may be very different than for adult animals (22). Alternative hypotheses regarding the mechanisms of metabolic feedback to the mitochondria have been presented elsewhere $(13,23,24)$.

Although the high energy phosphates remained stable through the level of tachycardia illustrated in Fig. 4, it was possible to cause changes in the PCr/ATP ratio by increasing heart rate to the point of hemodynamic compromise and decreased coronary blood flow (Fig. 5). Under these conditions of myocardial ischemia, as evidenced by the period of reactive hyperemia upon resuming the control heart rate, the decline of the $\mathrm{PCr} / \mathrm{ATP}$ ratio and recovery upon resuming the control heart rate are well within the time resolution of NMR spectroscopy. The time course of the fall in PCr/ATP when coronary flow (and presumably oxygen) was limiting is much more gradual than that predicted by an ADP, $P_{i}$ bireactant model (Fig. 6).

Pacing-induced coronary ischemia is already a useful clinical tool (25). In combination with ${ }^{31}$ P NMR measurements, it offers the opportunity to characterize in vivo myocardial energy metabolism under normal and pathologic conditions. The ability to follow cardiac high energy phosphates over the time course of a relatively brief metabolic stress appears to be a sensitive indicator of coronary supply/demand imbalance and could provide useful information to both basic scientists and clinicians. Although the time resolution attained under the invasive conditions of this study is not presently available for cardiac NMR studies in humans, steady state ${ }^{31} \mathrm{P}$ spectroscopy (i.e., several minutes acquisition time) are feasible (26). The insight provided into myocardial biochemistry in vivo may also serve as a measure of coronary insufficiency during stress testing.

In summary, the results of this study demonstrate that previous ${ }^{31} \mathrm{P}$ NMR findings during steady state conditions apply to the transient phase of changes in cardiac work. The left ventricular PCr/ATP ratio does not change sufficiently to explain tachycardia-induced increases in myocardial respiration, provided adequate myocardial blood flow can be maintained. When tachycardia resulted in a compromised coronary flow response the PCr/ATP ratio decreased significantly over $\sim 2$ min until a new steady state was reached. This effect was fully reversible within $\sim 1 \mathrm{~min}$ of resuming the original heart rate. These data indicate that: $(a)$ transient changes in PCr/ATP do not occur following an increase in $\mathrm{MVO}_{2}$ provided the coronary blood flow is not compromised. Thus, when coronary flow can respond appropriately to a metabolic stress whatever feedback mechanism serves to link cytosolic ATP hydrolysis with mitochondrial oxidative phosphorylation in the heart does so rapidly; $(b)$ factors other than the singular feedback of ATP hydrolysis products are involved in the regulation of myocardial respiration, and $(c)$ during conditions where myocardial supply/demand mismatching occurs there is a rapid decrease in PCr/ATP, suggesting that the $\mathrm{PCr} / \mathrm{ATP}$ ratio may be a useful marker of coronary ischemia in vivo.

\section{Appendix}

To estimate the time course and magnitude of the decrease in $\mathrm{PCr}$ and increase in $\mathrm{MVO}_{2}$ that would be expected if $\mathrm{ADP}$ and $\mathrm{P}_{\mathrm{i}}$ were regulating oxidative phosphorylation in response to a step change in the rate of ATP hydrolysis, the following model was constructed. Assuming equal contributions from ADP and $P_{i}$ to the feedback and Michaelis-Menten kinetics:

$\mathrm{MVO}_{2}=V_{\text {max }}$

$\times \frac{\left([\mathrm{ADP}] \times\left[\mathrm{P}_{\mathrm{i}}\right]\right) /(\operatorname{Kadp} \times K \mathrm{pi})}{1+[\mathrm{ADP}] / K \mathrm{Kadp}+\left[\mathrm{P}_{\mathrm{i}}\right] / K \mathrm{Kpi}+\left\{\left([\mathrm{ADP}] \times\left[\mathrm{P}_{\mathrm{i}}\right]\right) /(\operatorname{Kadp} \times K \mathrm{Ki})\right\}}$

where $[A D P]$ is calculated from the creatine kinase reaction (A2) and $K$ pi and $K$ adp are the in vitro $K_{\mathrm{m}}$ 's of $\mathrm{P}_{\mathrm{i}}$ and ADP, respectively. The in vitro $K_{\mathrm{m}}$ 's of $\mathrm{P}_{\mathrm{i}}(800 \mu \mathrm{M})$ and $\mathrm{ADP}(20-30 \mu \mathrm{M})$ have been previously determined (28-30) and are within the general range of the in vivo concentrations of $\mathrm{Pi}$ and ADP found in the canine myocardium (6). For this reason the initial [ADP] and $\left[\mathrm{P}_{\mathrm{i}}\right]$ were considered as being at their respective $K_{\mathrm{m}}$ 's, making the model conservative by maximizing the change in $\mathrm{MVO}_{2}$ for any given change in [ADP] or $\left[\mathrm{P}_{\mathrm{i}}\right] . V_{\max }$ was calculated from the resting values of $\mathrm{MVO}_{2}, \mathrm{ATP}, \mathrm{P}_{\mathrm{i}}$, and ADP using Eq. Al.

Equilibration of the creatine kinase reaction,

$[\mathrm{ATP}]=\left(\mathrm{Keq} \times[\mathrm{PCr}] \times[\mathrm{ADP}] \times\left[\mathrm{H}^{+}\right]\right) /[\mathrm{Cr}]$,

occurs much faster ( 10-fold) than ATP formation or breakdown (6). Keq for this reaction is $1.66 \times 10^{9}(31)$, the total creatine in the dog heart is $\sim 27.3 \mathrm{mM} \mathrm{(32)}$ (making [Cr] $\sim 27.3 \mathrm{mM}-[\mathrm{PCr}]$ ) and intracellular $\mathrm{pH}$ in the dog heart remains stable at 7.1 over a wide range of cardiac $\mathrm{MVO}_{2}$ 's (11). Hence, [PCr] is capable of buffering [ATP] far more rapidly than ATP is hydrolyzed by myosin ATPase. If the ATP hydrolysis in the myocardium at steady state is $\sim 5.6 \mathrm{~mol} / \mathrm{mol}$ of $\mathrm{O}_{2}$ consumed, the immediate change in ATP consumption at the myofibrils after a step change in cardiac work should approximate 5.6 times the difference between the initial $\mathrm{MVO}_{2}$ and the $\mathrm{MVO}_{2}$ once a new steady state is reached. The increase in $\mathrm{MVO}_{2}$ from 1.45 to 2.52 
$\mu \mathrm{mol} / \mathrm{g}$ per min seen with tachycardia associated with increased coronary flow would account for an increase in ATP hydrolysis of 6.0 $\mu \mathrm{mol} / \mathrm{g}$ per s. Due to the creatine kinase equilibrium [ATP] remains, for practical purposes, constant over this range of $\mathrm{MVO}_{2}$ and the ATP hydrolysis may be thought of as $\mathrm{PCr}$ hydrolysis. The resulting [PCr] at any given instant will be determined by the initial concentration, the amount of ATP hydrolyzed (expressed in terms of PCr via the creatine kinase reaction), the amount of ATP synthesized (again, in terms of $\mathrm{PCr}$ via creatine kinase) and the [ADP]. In other words,

$[\mathrm{PCr}]_{\mathrm{i}}=[\mathrm{PCr}]_{\mathrm{o}}+(\mathrm{PCr}$ formed $)-(\mathrm{PCr}$ hydrolyzed $)$

where the subscripts " $\mathrm{o}$ " and " $\mathrm{i}$ " designate starting and instantaneous conditions, respectively. Using the bireactant kinetics model (Eq. A1), the creatine kinase equilibrium (Eq. A2), the $\mathrm{MVO}_{2}$ at control and tachycardic heart rates (Table I), the initial PCr/ATP ratio and assuming that $\left[\mathrm{P}_{\mathrm{j}}\right]$ changes inversely to $[\mathrm{PCr}]$ on a mole for mole basis, it is possible to calculate the [PCr] (hence, the $\mathrm{PCr} / \mathrm{ATP}$ ratio), which could be expected at the higher steady state $\mathrm{MVO}_{2}$ if $A D P$ and $\mathrm{P}_{\mathrm{i}}$ were driving oxidative phosphorylation. By incrementing $[\mathrm{PCr}]$ between the initial and the predicted final steady state levels, the simultaneous [ADP], $\left[\mathrm{P}_{i}\right]$ and $\mathrm{MVO}_{2}$ can be calculated from the model for each [PCr]. This provides a solution for the transient phase of the step increase in ATP hydrolysis for the values of [PCr], [ADP], $\left[\mathrm{P}_{\mathrm{i}}\right]$ and $\mathrm{MVO}_{2}$, independent of the time course. The time course of the transient can then be calculated from the derivative of Eq. A3,

$\mathrm{d}[\mathrm{PCr}] / \mathrm{dt}=$ rate of $\mathrm{PCr}$ formation - rate of $\mathrm{PCr}$ breakdown

such that,

$\Delta t_{\mathrm{i}}=\frac{\Delta[\mathrm{PCr}]_{\mathrm{i}}}{\Delta(\mathrm{PCr} \text { formation })_{\mathrm{i}}-\Delta(\mathrm{PCr} \text { breakdown })_{\mathrm{i}}}$

where the rate of ( $\mathrm{PCr}$ formation) is determined from the rate of ATP formation via the creatine kinase equilibrium $\left(\mathrm{MVO}_{2 \mathrm{i}} \times 5.6 \sim \mathrm{ATP}\right.$ formation) and the rate of ( $\mathrm{PCr}$ breakdown $)_{\mathrm{i}}$ makes a step increase at $t$ $=0$ determined from the rate of ATP hydrolysis (ATP hydrolysis $\sim$ final steady state $\mathrm{MVO}_{2} \times 5.6$ ). Eq. A5 can be solved for $\Delta t$ by iteration, providing a time base for the previously determined values of $\mathrm{MVO}_{2}$ and $[\mathrm{PCr}]$

Using this model, the PCr/ATP ratio should decrease from the observed initial value of 1.55 to 1.24 (uncorrected for differential saturation) for the observed increase in $\mathrm{MVO}_{2}$ from 1.45 to $2.52 \mu \mathrm{mol} / \mathrm{g}$ per min (Table I). The time course following the transient in cardiac work is plotted in Fig. 6 . The time required for $>95 \%$ of the predicted fall in [PCr] to occur is $<20 \mathrm{~s}$ and the new steady state is attained in less than $1 \mathrm{~min}$. The expected change in [PCr] is well within the range of sensitivity of the statistics used in this study. Furthermore, this model was intentionally conservative in its primary assumptions. If ADP and $\mathrm{P}_{\mathrm{i}}$ are not near their $K_{\mathrm{m}}$ 's or do not contribute equally to regulation of respiration, the predicted decrease in the $[\mathrm{PCr}]$ for the observed change in $\mathrm{MVO}_{2}$ would be even greater.

\section{Acknowledgments}

The authors wish to thank Drs. Bruce A. Berkowitz, John Eng, and Teresa A. Fralix for helpful discussions and critical review of the manuscript.

\section{References}

1. Ackerman, J. J. H., T. H. Grove, G. C. Wong, D. G. Gadian, and G. K. Radda. 1980. Mapping of metabolites in whole animals by ${ }^{31} \mathrm{P}$ NMR using surface coils. Nature (Lond.). 283:167-170.

2. Balaban, R. S. 1984. The application of nuclear magnetic resonance to the study of cellular physiology. Am. J. Physiol. 246 (Cell. Physiol. 15):C10-C19.

3. Bittl, J. A., and J. S. Ingwall. 1985. Reaction rates of creatine kinase and ATP synthesis in the isolated heart. J. Biol. Chem. 260:3512-3517.

4. Clarke, K., and R. J. Willis. 1987. Energy metabolism and contractile function in rat heart during graded, isovolumic perfusion using ${ }^{31} \mathrm{P}$ nuclear magnetic resonance spectroscopy. J. Mol. Cell. Cardiol. 19:1153-1160.

5. Balaban, R. S., H. L. Kantor, L. A. Katz, and R. W. Briggs. 1986. Relation between work and phosphate metabolites in the in vivo paced mammalian heart. Science (Wash. DC). 232:1121-1123.

6. Katz, L. A., J. A. Swain, M. A. Portman, and R. S. Balaban. 1989. Relation between phosphate metabolites and oxygen consumption in heart in vivo. Am. J. Physiol. 256 (Heart Circ. Physiol. 25):H265-H274.

7. Opie, L. A. 1984. The Heart: Physiology, Metabolism, Pharmacology and Therapy. Grune \& Stratton, Inc., Philadelphia. 392 pp.

8. Stryer, L. 1988. Biochemistry. 3rd ed. W. H. Freeman, New York. 1089 pp.

9. Achs, M. J., D. Garkinkel, and L. A. Opie. 1982. Computer simulation of metabolism of glucose-perfused rat heart in a workjump. Am. J. Physiol. 243 (Regulatory Integrative Comp. Physiol. 12):R389-R399.

10. Portman, M. A., S. James, F. W. Heineman, and R. S. Balaban. 1988. Simultaneous monitoring of coronary blood flow and ${ }^{31} P$ NMR detected myocardial metabolites. Magn. Reson. Med. 7:243-247.

11. Katz, L. A., J. A. Swain, M. A. Portman, and R. S. Balaban. 1988. Intracellular pH and inorganic phosphate content of the heart in vivo: a ${ }^{31}$ P-NMR study. Am. J. Physiol. 255 (Heart Circ. Physiol. 24): $\mathrm{H} 189-\mathrm{H} 196$.

12. Kantor, H. L., R. W. Briggs, K. R. Metz, and R. S. Balaban. 1986. Gated in vivo examination of cardiac metabolites with ${ }^{31} \mathrm{P}$ nuclear magnetic resonance. Am. J. Physiol. 251 (Heart Circ. Physiol. 20):H171-H175.

13. Balaban, R. S., and F. W. Heineman. 1989. Control of mitochondrial respiration in the heart in vivo. Mol. Cell. Biochem. 89:191197.

14. Rushmer, R. F. 1976. Cardiovascular Dynamics. W. B. Saunders Co., Philadelphia. 584 pp.

15. Baller, D., A. Hoeft, H. Korb, H. G. Wolpers, J. Zipfel, and G. Hellige. 1981. Basic physiological studies on cardiac pacing with special reference to the optimal mode and rate after cardiac surgery. Thorac. Cardiovasc. Surg. 29:168-173.

16. Heineman, F. W., J. Eng, B. A. Berkowitz, and R. S. Balaban. 1989. NMR spectral analysis of kinetic data using natural lineshapes. Magn. Res. Med. 13:490-497.

17. Sokal, R. R., and F. J. Rohlf. 1969. Biometry. W. H. Freeman and Co., San Francisco. 776 pp.

18. Hochberg, Y., and A. C. Tamhane. 1987. Multiple Comparison Procedures. J. Wiley and Sons, New York. 450 pp.

19. Saito, D., K. Yasuhara, O. Nishiyama, S. Kusachi, and S. Haraoka. 1981. Comparative effects of heart rate and aortic blood pressure on MVO in the anesthetized open-chest dog. Jpn. Heart J. 22:833-837.

20. Kusachi, S., O. Nishiyama, K. Yasuhara, D. Saito, S. Haraoka, and H. Nagashima. 1982. Right and left ventricular oxygen metabolism in open-chest dogs. Am. J. Physiol. 243 (Heart Circ. Physiol. 12): $\mathrm{H} 761-\mathrm{H} 766$.

21. Katz, L. A., A. P. Koretsky, and R. S. Balaban. 1987. Respiratory control in the glucose perfused heart: a ${ }^{31} \mathrm{P}$ NMR and NADH fluorescence study. FEBS (Fed. Eur. Biochem. Soc.) Lett. 221:270276.

22. Portman, M. A., F. W. Heineman, and R. S. Balaban. 1989 Developmental changes in the relation between phosphate metabolites and oxygen consumption in the sheep heart in vivo. J. Clin. Invest. 83:456-464.

23. Groen, A. K., R. J. A. Wanders, H. V. Westerhoff, R. van der Meer, and J. M. Tager. 1982. Quantification of the contribution of various steps to the control of mitochondrial respiration. J. Biol. Chem. 257:2754-2757. 
24. Lemasters, J. J., R. Grunwald, and R. K. Emaus. 1984. Thermodynamic limits to the ATP/site stoichiometries of oxidative phosphorylation by rat liver mitochondria. J. Biol. Chem. 259:3058-3063.

25. Braunwald, E. 1988. Heart Diseases, 3rd ed. W. B. Saunders Co., Philadelphia. 1900 pp.

26. Bottomly, P. A. 1985. Non-invasive study of high energy phosphate metabolism in human heart using depth resolved ${ }^{31} \mathrm{P}$ NMR spectroscopy. Science (Wash. DC). 229:769-772.

27. Segel, I. H. 1975. Enzyme Kinetics. J. Wiley and Sons, New York. $957 \mathrm{pp}$.

28. Erecinska, M., and D. F. Wilson. 1982. Regulation of cellular energy metabolism. J. Membr. Biol. 70:1-14.

29. Ernster, L., C. Lee, and S. Jnada. 1966. The reaction sequence in oxidative phosphorylation. In Biochemistry of Mitochondria, E.
Slater, Z. Kaniuga, and L. Wojtczak, editors. Academic Press, London. p. 29-51.

30. Jacobus, W. E., R. W. Moreadith, and K. M. Vandegaer. 1982. Mitochondrial respiratory control: evidence against the regulation of respiration by extramitochondrial phosphorylation potentials or by ATP/ADP ratios. J. Biol. Chem. 257:2397-2402.

31. Larson, J. W. R., and R. L. Veech. 1979. Effects of pH and free $\mathrm{Mg}^{++}$on the $\mathrm{K}_{\text {eq }}$ of the creatine kinase reaction and other phosphate hydrolyses and phosphate transfer reactions. J. Biol. Chem. 254:6528-6537.

32. Witte, J., T. Ban, G. Hubner, O. Giersberg, E. Huegel, and H. J. Hirt. 1975. Metabolic and structural recovery of left ventricular canine myocardium from regional complete ischemia. Eur. Surg. Res. 7:136-155. 